\title{
Distribución y condiciones ambientales asociadas al comportamiento del delfín bufeo (Tursiops truncatus) y el delfín manchado (Stenella attenuata) (Cetacea: Delphinidae) en el Golfo Dulce, Costa Rica
}

\author{
Priscilla Cubero-Pardo \\ Fundación Promar. Apdo. 11709-1000, San José, Costa Rica. Dirección actual: Fundación Charles Darwin, Puerto \\ Ayora, Galápagos, Ecuador; pcubero@fcdarwin.org.ec
}

Recibido 30-V-2004. Corregido 25-VI-2006. Aceptado 12-I-2007.

\begin{abstract}
Distribution and environmental conditions related to the behavior in the bottlenose dolphin (Tursiops truncatus) and the spotted dolphin (Stenella attenuata) (Cetacea: Delphinidae) in Golfo Dulce, Costa Rica. Habitat characteristics influencing behavior in animal species vary locally. The influence that a particular environmental characteristic can have on a species depends not only on other variables, but on morphological, physiological and social conditions of that species. In this study, developed from June 1996 to July 1997, I studied whether specific behaviors are related to particular distribution areas and environmental factors in the bottlenose (Tursiops truncatus) and the spotted dolphin (Stenella attenuata). The study area was covered along oblicuous linear transects, and the behavior of single groups was observed from $15 \mathrm{~min}$ to $5 \mathrm{~h}$. Environmental factors such as depth, temperature, salinity and distance from shore, among others, were considered. For the bottlenose dolphin, foraging/feeding activities showed exclusive coincidence with river mouths, coral reef and mangrove areas, while social and milling activities where seen close to feeding areas. Traveling occurred along different points parallel to the coast, with a low percentage of cases across the gulf $(16.56 \%)$, suggesting that the bottlenose rarely crosses from one side to the other. In the spotted dolphin, several behaviors were observed simultaneously in the schools and it was not possible to associate areas with particular behaviors. The lack of significant relationships among activities and particular environmental variables (ANOVA tests) is attributed to three aspects: (a) transitions among activities generally occurred into a low variable area, (b) dolphins often traveled along large areas without changing activities and (c) environmental conditions in Golfo Dulce are homogeneous. In the two species the highest average in the number of individuals per group corresponded to the category of active socializing, followed by traveling, passive socializing and feeding. In the case of the bottlenose dolphin, the smallest group size was associated with feeding activities (ANOVA, $\mathrm{F}=2.624, \mathrm{p}=0.037$, $\mathrm{n}=156, \mathrm{df}=4$ ), while in the spotted dolphin the smallest group size corresponded to milling activities (ANOVA, $\mathrm{F}=3.817, \mathrm{p}=0.009, \mathrm{n}=51, \mathrm{df}=4$ ). Rev. Biol. Trop. 55 (2): 549-557. Epub 2007 June, 29.
\end{abstract}

Key words: environmental factors, behavior, Tursiops truncatus, Stenella attenuata, cetaceans, Costa Rica.

Todas las especies animales se asocian a características particulares de su hábitat, de tal forma que una interrelación de factores determina la forma como cada especie utiliza el ambiente que le rodea (Krebs 1985, Ferrero et al. 2002). Parece ser que en los cetáceos, factores tales como las tasas de depredación, la calidad y el espaciamiento de los parches de alimento, así como la facilidad con que dichos parches son encontrados, ejercen la mayor influencia en la estructura social de las distintas especies la cual, a su vez, influye grandemente en la forma como cada una utiliza su espacio (Pulliam y Caraco 1984, Mcleod et al. 2004).

Por su parte, las características del hábitat que influyen en el comportamiento varían de un sitio a otro. Estudios realizados con diferentes especies de cetáceos muestran que, en efecto, los factores ambientales que integran la ecología local influyen en ese aspecto de diversas maneras (Shane 1977, Norris y Dohl 1987, 
Shane 1990a, b, Ferrero et al. 2002, Mcleod et al. 2004).

En general, el comportamiento alimentario es el que ha recibido mayor análisis. Ha sido encontrada una relación entre éste y condiciones ambientales específicas tales como profundidad de la columna de agua (Shane 1990b), distancia de la costa (Shane 1977, Würsig y Würsig 1979), el flujo de la marea (Shane 1990b), las actividades humanas (Connor y Smolker 1985, Corkeron et al. 1990) y el tipo de sedimento (Mcleod et al. 2004). Würsig y Würsig (1979) encontraron que las actividades de descanso en el bufeo (Tursiops truncatus, Montagu, 1821) eran realizadas cerca de la costa temprano en la mañana. En el Neotrópico, hay muy poca información al respecto.

Este estudio se enfocó en el objetivo de determinar si las distintas actividades realizadas por los delfines bufeo y manchado (Stenella attenuata, Gray, 1846) se encuentran en zonas específicas dentro de sus respectivas áreas de distribución y si a cada actividad se asocian características ambientales particulares. Se esperaba encontrar asociaciones específicas entre cada tipo de comportamiento y las áreas donde ocurren, así como asociaciones particulares entre variables ambientales y cada categoría mayoritaria de comportamiento.

\section{MATERIALES Y MÉTODOS}

Área de estudio: Golfo Dulce ( $08^{\circ} 30^{\prime} \mathrm{N}$, $83^{\circ} 16^{\prime} \mathrm{W}$ ) es considerado un ecosistema único a lo largo de la costa pacífica de Costa Rica, pues es definido como un fiordo tropical debido a sus características físico-químicas (Richards et al. 1971, Hebblen et al. 1996). El Golfo Dulce tiene bajos niveles de productividad en comparación con el océano abierto y golfos verdaderos (von Wangelin y Wolff 1996). A pesar de este hecho, este ecosistema sostiene una alta diversidad de organismos incluyendo aves y tortugas marinas, serpientes, invertebrados y varias especies de peces y reúne, junto con las aguas que rodean la Península de Osa, un total de 22 especies de cetáceos (J. Rodríguez-
Fonseca, com. pers.), de 28 documentadas para Costa Rica (Rodríguez-Fonseca 2001).

Frecuencia de avistamientos: el estudio se extendió desde julio de 1996 hasta junio de 1997 e incluyó dominancia de avistamientos de delfín bufeo como se describe en Cubero-Pardo (1999).

Método de muestreo: el área de estudio fue cubierta como se describe en Cubero-Pardo (1999), siguiendo transectos lineares oblicuos pre-determinados. Los avistamientos considerados para el análisis abarcaron ámbitos de observación de 15 min hasta $5 \mathrm{~h}$, e implicaron un solo grupo a la vez. Al inicio de cada avistamiento y al término de cada hora dentro del mismo fueron medidas variables ambientales, establecida la posición geográfica con el uso de un navegador portátil y clasificado el comportamiento en categorías discretas y divididas en alimentación, merodeo, desplazamiento, socialización activa y socialización pasiva (CuberoPardo 1999).

Factores ambientales: fue medida la temperatura superficial del agua $\left({ }^{\circ} \mathrm{C}\right)$ utilizando un termómetro digital, la salinidad (\%o) utilizando un refractómetro y la profundidad (m) con base en un mapa batimétrico. Asimismo, fueron estimados la velocidad del viento $(\mathrm{km} / \mathrm{h})$, el porcentaje de cobertura nubosa, el oleaje (m) y el estado de la superficie del mar, en cuyo caso fue utilizada la escala de Beaufort, donde 1 implicó ausencia total de ondas; 2 reflejó presencia de pequeñas ondas sin espuma; 3 incluyó formación de espuma en algunas crestas, y 4 la formación de crestas con rompimiento y más espuma. La distancia respecto a la costa fue estimada en cada caso con base en un valor conocido, dado por el sistema de navegación portátil respecto a un punto previamente programado.

Determinación del comportamiento: el comportamiento fue clasificado dentro de cinco categorías discretas, como se describe en Cubero-Pardo (1999). Para todo efecto, un grupo fue definido como cualquier número 

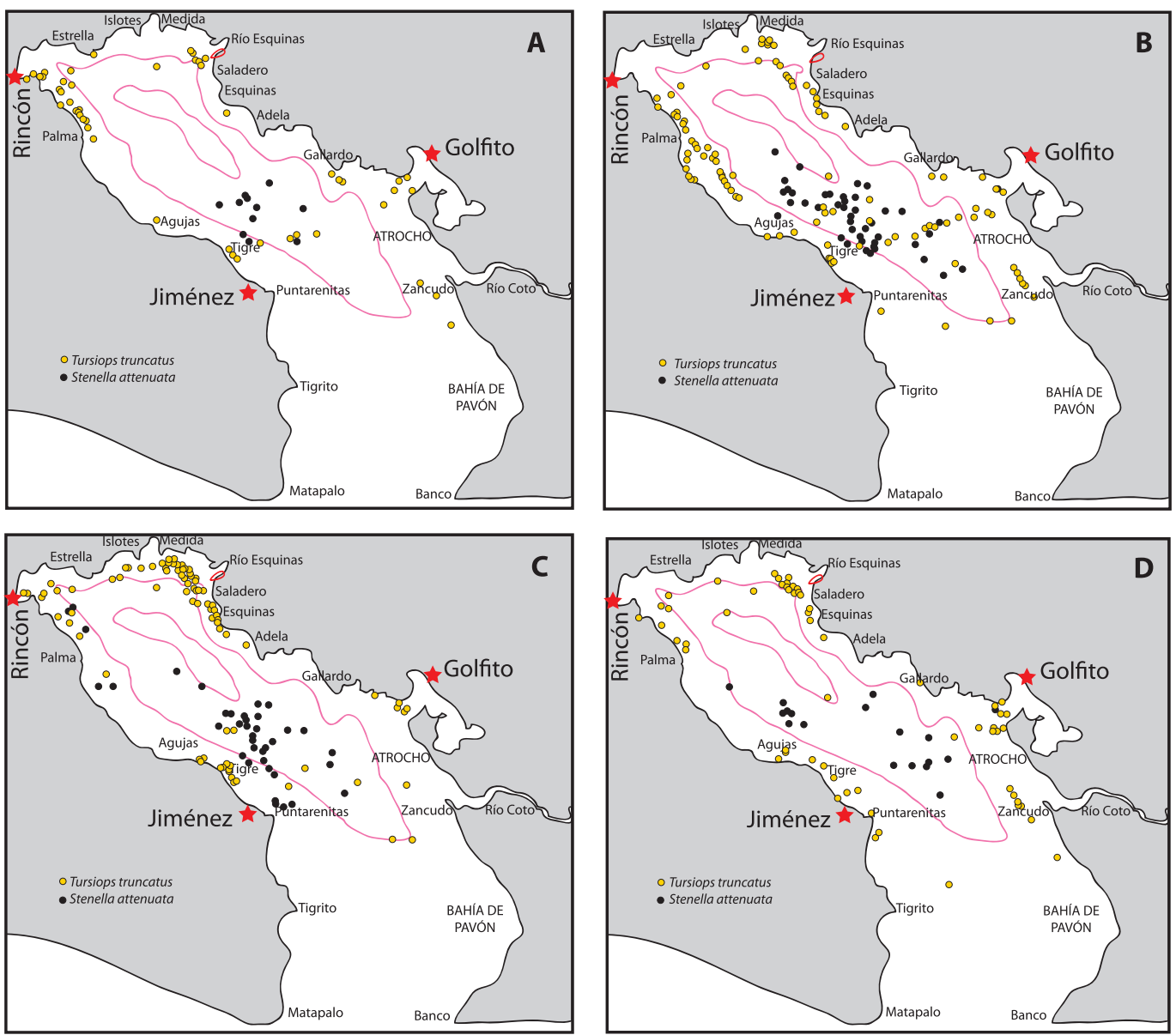

Fig. 1. Áreas de distribución asociadas con el comportamiento alimentario (a), de desplazamiento (b), social (c) y de merodeo (d) del bufeo (puntos claros) y el delfín manchado (puntos oscuros) en el Golfo Dulce. Julio 1996-Junio 1997.

Fig. 1. Distribution areas associated with feeding behavior (a), traveling (b), social behavior (c) and milling (d) of the bottlenose dolphin (clear points) and the pantropical spotted dolphin (dark points) in Golfo Dulce. July 1996-June 1997.

de delfines observado en asociación aparente, moviéndose en la misma dirección y a menudo, pero no siempre, involucrado en la misma actividad (Shane 1990b).

Análisis estadísticos: la relación entre los factores ambientales y las categorías de comportamiento para ambas especies fue explorada mediante un ANDEVA de bloques. La misma prueba fue utilizada para determinar la relación del tamaño de grupo con la actividad.

\section{RESULTADOS}

\section{Distribución según las categorías de comportamiento}

\section{Bufeo}

Alimentación: en la costa occidental, las actividades de alimentación ocurrieron principalmente en la boca de los ríos Rincón, Agujas y Tigre, así como en áreas limítrofes 
al manglar entre Bahía Rincón y Punta Palma. En la costa oriental, la alimentación ocurrió en la desembocadura de los ríos Esquinas y Coto Colorado y, en los arrecifes rocosos cercanos a Punta Gallardo, en cuyo caso los bordes costeros consisten en paredones casi verticales cubiertos de vegetación arbórea y en la entrada a Golfito (Fig. 1A).

Desplazamiento: fueron encontrados desplazándose a lo largo de la costa occidental, desde Punta Tigre hasta Bahía Rincón, tanto en dirección norte como sur; a lo largo de la costa norte, desde Bahía Rincón hasta punta Esquinas, en direcciones noroeste y noreste y cruzando el golfo desde la entrada a Golfito hacia Puerto Jiménez y desde Río Agujas hacia Punta Gallardo (Fig. 1B).

En $16.56 \%(n=42)$ de las veces se encontró al delfín bufeo en el centro del golfo, desplazándose de un lado a otro. En $23.8 \%$ del inicio de los avistamientos los delfines parecieron provenir del centro, luego de lo cual continuaron moviéndose en dirección paralela a la costa o se establecieron en un área definida donde iniciaron una nueva actividad.

Socialización: los delfines fueron vistos socializando en Bahía Rincón; desde Los Mogos hasta Punta Esquinas, en el lado norte; entre Punta Agujas y Punta Tigre, en el lado occidental, y cerca del centro, entre Río Tigre y Río Coto Colorado (Fig. 1C).

Merodeo: las actividades de merodeo ocurrieron en las costas oriental y occidental. En la primera fue común encontrar al bufeo merodeando cerca de la desembocadura del Río Coto Colorado, en la entrada y dentro de Golfito y en la desembocadura del Río Esquinas. En la costa occidental, las actividades de merodeo se dieron entre Puntarenitas y Punta Agujas, y entre Bahía Rincón y Punta Palma (Fig. 1D).

\section{Manchado}

El delfín manchado tendió a ubicarse en o cerca del centro (Cubero-Pardo 1998) y cuando se desplazó lo hizo sobre todo con tendencia a estar más cerca de la costa occidental del golfo, a una distancia aproximada entre $\operatorname{los} 2000$ y 8 000 m (Fig. 3A). Su movilización prácticamente constante durante todos los avistamientos fue tan variable en dirección que no fue posible hallar tendencias con relación al comportamiento.

Debido a que esta especie se agrupó generalmente formando una manada (Cubero-Pardo 1998), los distintos grupos que la componían, por lo general, no realizaron la misma actividad. Por ejemplo, mientras uno o más grupos se dedicaban a actividades de alimentación, otros realizaban interacciones sociales y otros simplemente merodeaban. Por esto, en general, las áreas de distribución asociadas a las diferentes actividades no fueron diferenciales (Fig. 1A, D).

\section{Características del grupo según la actividad}

Tamaño de grupo: en ambas especies el mayor promedio en el número de individuos por grupo correspondió a la categoría de socialización activa, seguido en forma decreciente por el desplazamiento, la socialización pasiva y la alimentación. Sólo en el caso del merodeo hubo diferencias entre ambas especies en la ubicación del tamaño de grupo en relación con los demás comportamientos. En el caso del bufeo, el menor tamaño promedio de grupo estuvo asociado a las actividades de alimentación (ANDEVA, $\mathrm{F}=2.624, \mathrm{p}=0.037, \mathrm{gl}=4$ ), mientras que en el manchado correspondió a los momentos de merodeo (ANDEVA, $\mathrm{F}=$ 3.817, $\mathrm{p}=0.009, \mathrm{gl}=4$ ) (Fig. 2).

\section{Factores ambientales asociados a los distintos tipos de comportamiento}

Ninguna de las variables siguientes mostró diferencias estadísticamente significativas entre categorías de comportamiento; sin embargo, las tendencias observadas revelan resultados interesantes que se describen a continuación:

Distancia respecto a la costa: tanto en el caso del bufeo como del delfín manchado, el 

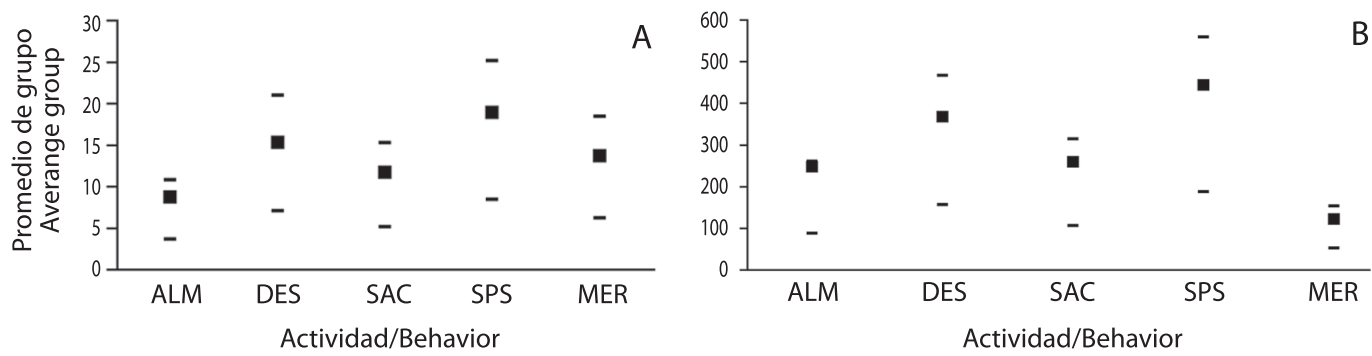

Fig. 2. Tamaño promedio de grupo según la categoría de comportamiento (ALM: alimentación; DES: desplazamiento; SAC: socialización activa; SPS: socialización pasiva y MER: merodeo) para el bufeo (a) y el delfín manchado (b) en el Golfo Dulce. Julio 1996-Junio 1997. Las barras de error representan intervalos de confianza del 95\%.

Fig. 2. Average group size according to behavioral category (ALM: feeding; DES: traveling, SAC: active socializing; SPS: passive socializing and MER: milling) for the bottlenose dolphin (a) and the pantropical spotted dolphin (b) in Golfo Dulce. July 1996-June 1997. Error bars represent confidence intervals of 95\%.

promedio de distancia menor correspondió a las actividades de alimentación; mientras que el mayor promedio de distancia respecto a la costa quedó referido a las actividades de socialización activa, en el caso del delfín bufeo (Fig. 3A), y a la socialización pasiva, en el caso del delfín manchado (Fig. 3B).

Profundidad: en ambas especies, las actividades de alimentación, desplazamiento y merodeo coincidieron con áreas menos profundas en comparación con las actividades sociales. Las actividades de alimentación y de merodeo se asociaron a las zonas de menor profundidad en el bufeo, mientras que en el delfín manchado tal condición se asoció al desplazamiento y al merodeo (Fig. 3).

La menor salinidad se asoció a las áreas de alimentación y merodeo en el caso del bufeo, mientras que para el delfín manchado esas actividades fueron realizadas en áreas con la mayor salinidad respecto a las otras actividades (Fig. 3).

Temperatura: las áreas de alimentación del bufeo coincidieron con la menor temperatura promedio en comparación con sus demás actividades. Caso contrario ocurrió con el delfín manchado, para el cual las actividades de alimentación estuvieron relacionadas a zonas con la mayor temperatura promedio respecto a otras actividades. La diferencia de temperatura entre las áreas de forrajeo de ambas especies fue de poco más de un grado (Fig. 3).

Oleaje: para el bufeo, las condiciones de oleaje fueron mínimas durante las actividades de alimentación y merodeo y máximas durante las actividades sociales. En términos generales, la misma situación se dio en el caso del delfín manchado, excepto porque las actividades de merodeo coincidieron con el mayor oleaje promedio respecto a las otras actividades (Fig. 3).

No fueron encontradas tendencias claras en relación conl resto de las variables ambientales registradas. Las condiciones en tales casos fueron, en general, muy uniformes (CuberoPardo 1998).

\section{DISCUSIÓN}

Las figuras de distribución según las actividades muestran traslapes que explican, al menos en parte, la falta de significancia estadística en lo que a interacción de condiciones ambientales se refiere. Cubero-Pardo (1999) evidencia que, además de la existencia de patrones de actividad claramente definidos en ambas especies, cada una presenta secuencias 
A
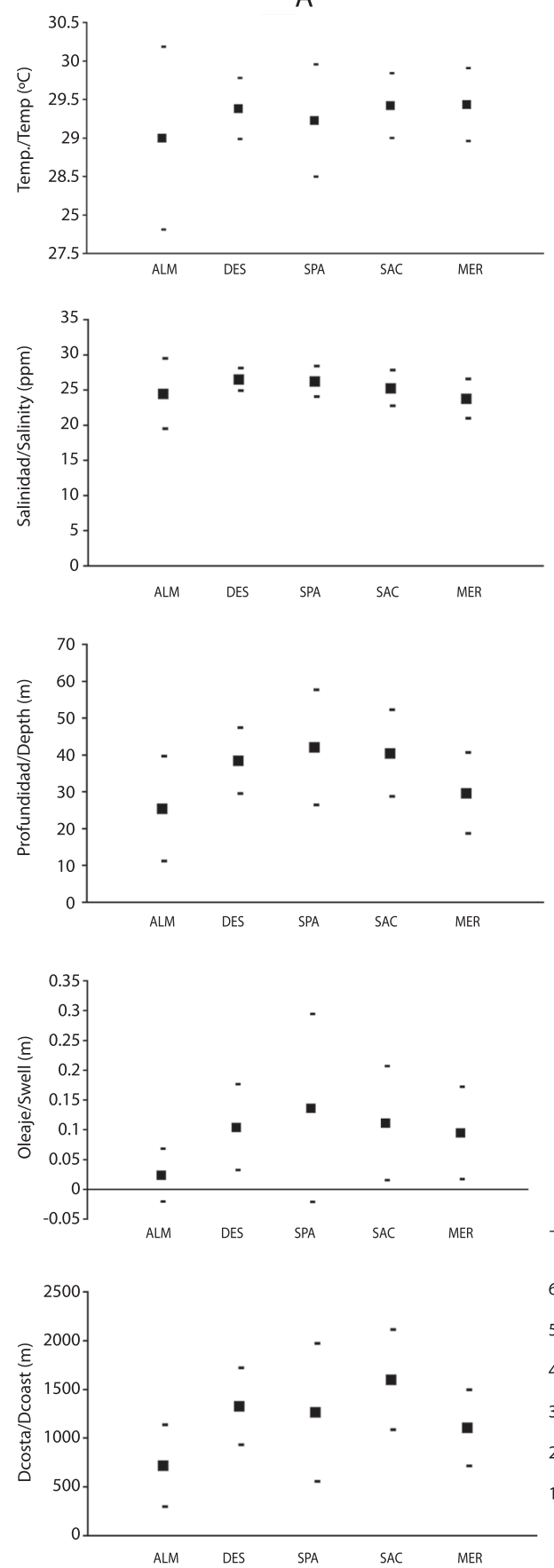

Actividad/Behavior
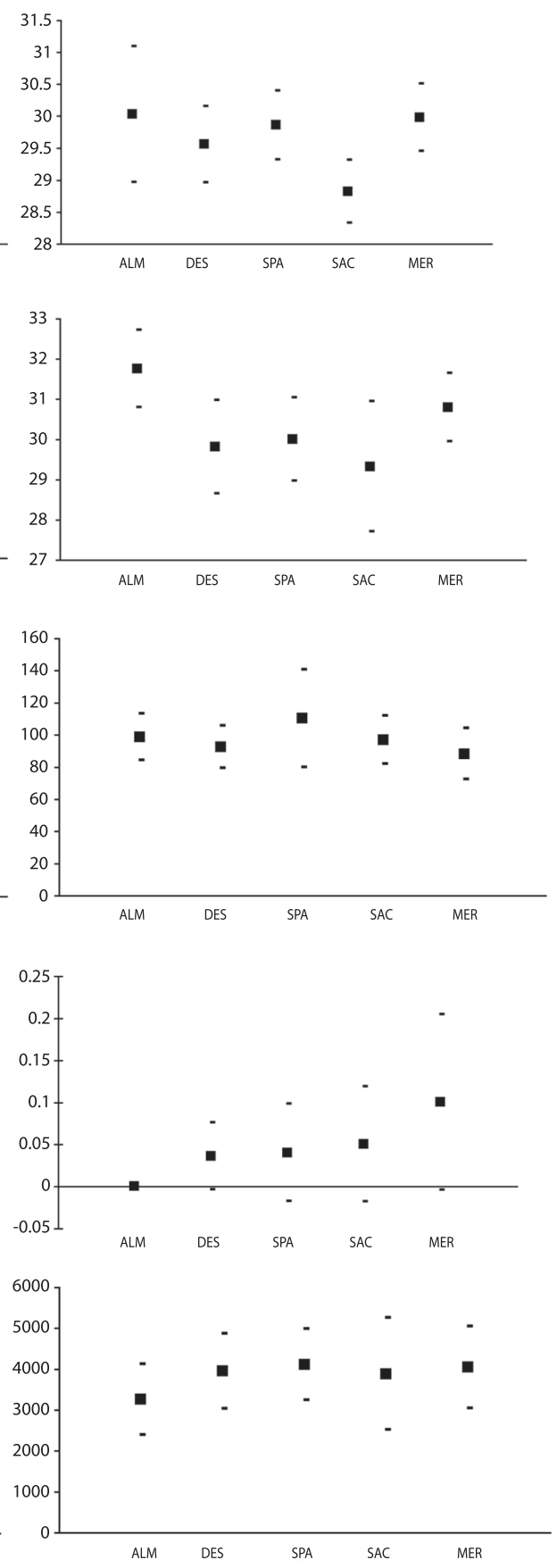

Actividad/Behavior

Fig. 3. Valores promedio de factores ambientales según la actividad para el bufeo (a) y el delfín manchado (b) en el Golfo Dulce, con intervalos de confianza del 95\%. Julio 1996-Junio 1997.

Fig. 3. Average values of environmental factors according to behavior for the bottlenose dolphin (a) and the pantropical spotted dolphin (b) in Golfo Dulce, with confidence intervals of 95\%. July 1996 to June 1997. 
o transiciones en un continuo de un comportamiento a otro. Dichas transiciones ocurren sin mayor cambio de posición o, por el contrario, con demasiada movilidad.

Las condiciones ambientales consideradas, a excepción de la profundidad, resultan bastante uniformes en términos generales en el Golfo Dulce (Cubero-Pardo 1998). Sin embargo, las áreas de forrajeo fueron claramente definibles tanto dentro de cada especie como entre especies. Condiciones de menor salinidad y temperatura en las áreas de forrajeo del bufeo, sugieren diferencias en las preferencias de hábitat de las presas consumidas por ambas especies de delfines. Cambios físicos sutiles pueden afectar significativamente a los peces según sus capacidades fisiológicas (Lagler et al. 1977).

En este estudio, la asociación de las actividades de alimentación de los bufeos con las desembocaduras de los ríos fue lo más común, en términos generales. Aunque el Golfo Dulce se caracteriza por una baja productividad en comparación con zonas oceánicas (von Wangelin y Wolff 1996), las áreas de desembocadura son las más productivas, especialmente la del Río Coto Colorado (A. Morales, com. pers.). Adicionalmente, la asociación de los bufeos con desembocaduras de ríos es una situación bastante común en diferentes partes del planeta (Leatherwood y Reeves 1983, Jefferson et al. 1994).

Las actividades de desplazamiento cubrieron la mayor área, lo cual debió ser resultado de que en muchas ocasiones ocurrían junto con las otras actividades, así como de la naturaleza de la actividad en sí misma. Además, las actividades de desplazamiento presentaron el mayor traslape espacial, aunque no temporal, entre especies. En el bufeo, la socialización mostró coincidencia con las desembocaduras de los ríos y partes adyacentes. En gran medida, esto debió tener relación con la mayor tendencia a realizar actividades sociales poco después de alimentarse, sin cambiar significativamente de posición (Cubero-Pardo 1999).

La menor distancia respecto a la costa asociada a las actividades de alimentación en ambas especies, pudo reflejar una distribución de presas tendientes a estar relativamente cerca de la costa como una forma de aprovechar mayor concentración de sus propias presas. Contrariamente, las actividades sociales ocuparon las posiciones más alejadas de la costa en ambos casos, pero en lo que respecta al bufeo, la socialización activa fue la que ocupó la posición relativamente más lejana. Esto, dentro de las características de su área de distribución preferente significa mayor profundidad, lo cual pudo brindar espacio tridimensional necesario para realizar sus exhibiciones (K. Dudzinsky, com.pers.).

El menor oleaje en las zonas de alimentación debió asociarse a la hora del día en que dichas actividades ocurrieron en ambas especies. Mientras, en el caso del bufeo, la presencia de oleaje estuvo mayormente asociada con la realización de actividades sociales, el efecto totalmente opuesto se notó en el delfín manchado, el cual tendió hacia el merodeo. Estas diferencias pudieron estar asociadas a los patrones de actividad en sí mismos.

El menor número de individuos asociado a actividades de alimentación en el caso del bufeo reflejó su tendencia a aprovechar estrategias de forrajeo individualizadas, caso contrario del delfín manchado (A. Acevedo, com. pers.) El menor tamaño de grupo asociado a la socialización activa respecto a la forma pasiva en ambas especies, se debió a que el primero involucra acciones sumamente cercanas entre los individuos, incluyendo actividad sexual (Dawson y Slooten 1996), las cuales se mantienen por largos períodos de tiempo de tal modo que el reconocimiento de unos a otros para fines cooperativos sea exitoso (Würsig et al. 1991). Además, el comportamiento social pasivo es dirigido especialmente a interacciones con la embarcación (Cubero-Pardo 1999), no a interacciones entre los individuos mismos, lo cual parece indicar un estado de mayor independencia.

El desplazamiento es usualmente explicado como una estrategia de evasión de depredadores (Shane et al. 1986). El mayor número de individuos promedio por grupo encontrado 
en dicha actividad, sumado a la tendencia a la menor distancia respecto a la costa en ambas especies, podría reflejar formas adicionales de protección a los cambios de lugar.

Es posible concluir que la homogeneidad superficial de los factores ambientales abióticos del golfo, sumado a la falta de efecto directo de los mismos sobre los delfines, así como la alta movilidad que ésos presentan dentro de sus conspicuas áreas de distribución, limitan las posibilidades de que exista una asociación marcada entre actividades y áreas con condiciones particulares. Sin embargo, condiciones dadas de temperatura y salinidad se relacionan con las actividades de alimentación en ambas especies, mientras que condiciones de profundidad y oleaje pueden beneficiar la presencia de actividades sociales.

\section{AGRADECIMIENTOS}

Agradezco profundamente a mi padre Álvaro Cubero Rodríguez ${ }^{\dagger}$, por su inmenso apoyo en el desarrollo de la investigación original de la cual se extrae esta publicación. Gracias también a la Fundación para la Investigación de la Universidad, a la Vicerrectoría de Investigación de la Universidad de Costa Rica, a la Empresa Borda Azul y al Instituto Costarricense de Pesca y Acuacultura, por el apoyo económico y logístico brindados.

\section{RESUMEN}

Las características del hábitat que influyen en las especies animales varían de un lugar a otro. Al mismo tiempo, toda especie es rodeada por una serie de factores ambientales que interactúan entre sí, pero la influencia que una característica ambiental particular pueda ejercer sobre una especie depende no sólo de las demás variables, sino de las condiciones morfológicas, fisiológicas y sociales de esa especie. En este estudio, desarrollado de junio 1996 a julio 1997, la pregunta fue enfocada a determinar si comportamientos específicos en el delfín bufeo (Tursiops truncatus) y el delfín manchado (Stenella attenuata) están relacionados con áreas de distribución y factores ambientales particulares. El área de estudio fue cubierta siguiendo transectos lineares oblicuos predeterminados y cada grupo fue observado de $15 \mathrm{~min}$ a 5 h. Las variables ambientales fueron medidas al inicio y al final de cada avistamiento junto con la determinación de la posición geográfica y el comportamiento llevado a cabo por los delfines. Para el bufeo, las actividades de forrajeo/ alimentación mostraron coincidencia exclusiva con bocas de ríos y áreas de coral y manglar, y las actividades sociales y de merodeo, se asociaron a áreas cercanas a los sitios de forrajeo. El desplazamiento ocurrió a lo largo de diferentes puntos paralelos a la costa, con un bajo porcentaje de casos a través del golfo ( $16.56 \%$ ), lo cual sugiere que el bufeo rara vez cruza el golfo. En el caso del manchado, no fue posible asociar zonas comportamientos particulares, pues todo el grupo se mueve junto; sin embargo, si fue observada la presencia de distintos comportamientos en un mismo momento, llevados a cabo por grupos específicos dentro de la manada. Se consideraron factores ambientales tales como la profundidad, la temperatura, la salinidad y la distancia respecto a la costa, entre otros. El ANDEVA de bloques no reveló una relación marcada entre actividades y zonas variables ambientales particulares, sin embargo, los resultados mostraron información valiosa sobre tendencias muy interesantes. La falta de significancia, en relación con los aspectos ambientales, fue atribuida a tres aspectos: (a) las transiciones entre actividades se dieron generalmente dentro de un área poco variable, (b) fue común que los delfines se desplazaran cubriendo grandes áreas sin cambiar de actividad y (c) las condiciones ambientales en el Golfo son bastante homogéneas. En ambas especies el mayor promedio en el número de individuos por grupo correspondió a la categoría de socialización activa, seguido en forma decreciente por el desplazamiento, la socialización pasiva y la alimentación. En el caso del bufeo, el menor tamaño promedio de grupo estuvo asociado a las actividades de alimentación (ANDEVA, $\mathrm{F}=2.624, \mathrm{p}=0.037, \mathrm{gl}=4$ ), mientras que en el manchado correspondió a los momentos de merodeo (ANDEVA, $\mathrm{F}=3.817, \mathrm{p}=0.009, \mathrm{gl}=4$ ).

Palabras clave: factores ambientales, comportamiento, Tursiops truncatus, Stenella attenuata, cetáceos, Costa Rica.

\section{REFERENCIAS}

Connor, R.C. \& R.S. Smolker. 1985. Habituated dolphins (Tursiops sp.) in Western Australia. J. Mammal. 66: 398-400.

Corkeron, P.J., M.M. Bryden \& K.E. Hedstrom. 1990. Feeding by bottlenose dolphins in association with trawling operations in Moreton Bay, Australia, p. 329-336. In S. Leatherwood \& R.R. Reeves (eds.). The bottlenose dolphin. Academic, San Diego, California, EEUU. 
Cubero-Pardo, P. 1998. Distribución y patrones de actividad del bufeo (Tursiops truncatus) y el delfín manchado (Stenella attenuata) en el Golfo Dulce, en relación con variables ambientales. Tesis de maestría, Universidad de Costa Rica, San José, Costa Rica. $102 \mathrm{p}$.

Cubero-Pardo, P. 1999. Patrones de comportamiento diurnos y estacionales de Tursiops truncatus y Stenella attenuata (Mammalia: Delphinidae) en el Golfo Dulce, Costa Rica. Rev. Biol. Trop. 46: 103-110.

Dawson, S.M. \& E. Slooten. 1996. Down-under dolphins: the story of Hector's dolphin. Canterburry University, Christchurch, Nueva Zelanda. 60 p.

Ferrero, R.C., R.C. Hobbs \& G.R. VanBlaricom. 2002. Indications of habitat use patterns among small cetacenas in the central North Pacific based on fisheries observer data. J. Cetacean Res. Manage. 4: $311-321$.

Hebblen, D., D. Beese \& J. Cortés. 1996. Morphology and sediment structures in Golfo Dulce, Costa Rica. Rev. Biol. Trop. 44: 1-10.

Jefferson, T.A., S. Leatherwood \& M.A. Webber. 1994. FAO species identification guide. Marine Mammals of the World. FAO, Roma, Italia. 320 p.

Krebs, C.J. 1985. Ecología: Estudio de la distribución y la abundancia. Harla, México DF, México. 753 p.

Lagler, K.F, J.E. Bardach, R.R. Miller \& D.R. May Passino. 1977. Ictiología. AGT, México DF, México. 489 p.

Leatherwood, S. \& R.R. Reeves. 1983. The Sierra Club Handbook of Whales and dolphins. Sierra Club, San Francisco, EEUU. 302 p.

Mcleod, K., R. Fairbairns, A. Gill, B. Fairbairns, J. Gordon, C. Blair-Mayers \& E.C.M. Parsons. 2004. Seasonal distribution of minke whales Balaenoptera acutorostrata in relation to physiography and prey off the isle of Mull, Scotland. Mar. Ecol. Prog. Ser. 277: 263-274

Norris, K.S. \& T.P. Dohl. 1987. The behavior of the Hawaiian spinner porpoise, Stenella longirostris. Fish. Bull. 77: 821-847.
Pulliam H.R. \& T. Caraco. 1984. Living in groups: is there an optimal group size? p. 122-147. In J.R. Krebs \& N.B. Davies (eds.). Behavioural ecology, an evolutionary approach. Blackwell, Oxford, Reino Unido.

Richards, F.A., J.J. Anderson \& J.D. Cline. 1971. Chemical and physical observations in Golfo Dulce, an anoxic basin in the Pacific coast of Costa Rica. Limnol. Oceanogr. 16: 43-50.

Rodríguez-Fonseca, J. 2001. Diversidad y distribución de los cetáceos de Costa Rica (Cetacea: Delphinidae, Physeteridae, Ziphiidae y Balaenopteridae). Rev. Biol. Trop. 49: 135-143.

Shane, S.H. 1977. The population biology of the atlantic bottlenose dolphin, Tursiops truncatus, in the Aransas Pass area of Texas. M.Sc. Thesis, Texas A\&M University, College Station, Texas, EEUU. 239 p.

Shane, S.H. 1990a. Comparison of bottlenose dolphin behavior in Texas and Florida, with a critique of methods for studying dolphin behavior, p. 541-558. In S. Leatherwood \& R.R. Reeves (eds). The bottlenose dolphin. Academic, San Diego, California, EEUU.

Shane, S.H. 1990b. Behavior and Ecology of the bottlenose dolphin at Sanibel Island, Florida, p. 245-265. In S. Leatherwood \& R.R. Reeves (eds.). The bottlenose dolphin. Academic, San Diego, California, EEUU.

Shane, S.H., R.S. Wells \& B.Würsig. 1986. Ecology, Behavior and Social organization of the bottlenose dolphin: a review. Mar. Mamm. Sci. 2: 34-63.

von Wangelin, M. \& M. Wolff. 1996. Comparative biomass spectra and species composition of the zooplancton communities in Golfo Dulce and Golfo de Nicoya, Pacific coast of Costa Rica. Rev. Biol. Trop. 44: 135-155.

Würsig, B. \& M. Würsig. 1979. Behavior and ecology of the bottlenose dolphin, Tursiops truncatus, in the South Atlantic. Fish. Bull. 77: 339-412.

Würsig, B., F. Cipriano \& M. Würsig. 1991. Dolphin movement patterns: information from radio and theodolite tracking studies, p. 79-111. In K. Pryor \& K.S. Norris (eds.). Dolphin Societies: discoveries and puzzles. University of California, California, EEUU. 
